\title{
Comparação dos programas TC2DFTPL e TCFOUR para o cálculo da correção do terreno no Estado do Rio Grande do Sul
}

Gabriela Pasetto Falavigna, Sérgio Florêncio de Souza, Silvia Beatriz Alves Rolim. Universidade Federal do Rio Grande do Sul (UFRGS).

Copyright 2014, SBGf - Sociedade Brasileira de Geofísica

Este texto foi preparado para a apresentação no VI Simpósio Brasileiro de Geofísica, Porto Alegre, 14 a 16 de outubro de 2014. Seu conteúdo foi revisado pelo Comitê Técnico do VI SimBGf, mas não necessariamente representa a opinião da SBGf ou de seus associados. É proibida a reprodução total ou parcial deste material para propósitos comerciais sem prévia autorização da SBGf.

\section{Resumo}

A correção de terreno é uma das correções aplicadas ao valor da aceleração da gravidade. O objetivo desse trabalho consistiu na comparação do desempenho dos programas TC2DFTPL e TCFOUR utilizados no cálculo da correção topográfica para o Estado do Rio Grande do Sul. Os resultados obtidos para os programas TC2DFTPL e TCFOUR são iguais. Notouse uma incompatibilidade entre as escalas de variação da correção do terreno dos modelos derivados do SRTM e do ASTER com resoluções de $2 \mathrm{~km}$ e $1 \mathrm{~km}$ calculados pelos dois programas. Concluiu-se que essa incompatibilidade se deva à necessidade de compartimentar a área de estudo para as maiores resoluções e também às diferenças altimétricas entre os dois modelos digitais do terreno, que são mais evidentes nas regiões de maiores altitudes.

\section{Introdução}

O efeito gravitacional das massas topográficas, localizadas acima do geóide, deve ser considerado em várias aplicações da Geodésia Física, como, por exemplo, no cálculo das anomalias da gravidade (anomalia free-air, de Bouguer, isostática) e das ondulações do geóide (Matos, 2005).

A anomalia free-air é obtida através da aplicação da correção free-air, que considera a variação da gravidade entre a superfície física e a superfície do geóide, utilizando a altitude do ponto e desconsiderando a massa entre essas superfícies. A anomalia de Bouguer é obtida a partir da correção Bouguer, que remove o efeito das massas entre a estação e o geóide, assumindo que 0 espaço entre a superfície física e a superfície do geóide seja preenchido uniformemente com massa, no caso de uma estação situada acima do geóide e uniformemente vazio, se estiver abaixo do geóide. A anomalia isostática leva em conta a correção topo-isostática, a qual também considera a heterogeneidade de massa abaixo do geóide, entendida como massa de compensação da topografia acima do geóide (Jamur et. al., 2010).

A determinação do geóide através de dados gravimétricos envolve a solução do Problema do Valor do Contorno da Geodésia (PVCG), o qual pressupõe duas condições (Heiskanen; Moritz, 1967; Gemael, 1999, apud
Matos, 2005): as medidas gravimétricas devem ser feitas sobre a superfície geoidal; e não devem existir massas externas ao geóide.

A primeira condição é satisfeita através da correção free-air, que reduz o valor observado da gravidade ao geóide; e a segunda, pode ser atendida através do método de condensação de Helmert (Lambert, 1930, apud Matos, 2005), por meio do qual as massas topográficas são removidas e subsequentemente recolocadas no interior do geóide, considerando as massas externas com uma densidade específica.

A correção do terreno (CT) é uma das reduções aplicadas à aceleração da gravidade. Com a aplicação da correção do terreno nas anomalias gravimétricas, as massas externas ao geóide são verticalmente comprimidas sob a superfície do geóide, alterando o potencial gravitacional da Terra. Atualmente, o cálculo dessa correção, tem sido possível, de forma sistemática, com a utilização de modelos digitais do terreno (MDTs) locais ou globais (Jamur et. al., 2010), pois as elevações são fornecidas em grades de diferentes tamanhos que proporcionam o uso dos modelos topográficos de massa linear e de prismas retangulares, através do uso da FFT (Fast Fourrier Transform) na fórmula da correção do terreno.

Nesse trabalho, utilizaram-se os programas TC2DFTPL e TCFOUR juntamente com os dados provenientes dos MDTs do SRTM e do ASTER GDEM para o cálculo da correção de terreno a ser aplicada nas observações gravimétricas do Estado do Rio Grande do Sul.

A área de estudo para a realização desse trabalho situa-se na porção sul do Brasil e corresponde ao Estado do Rio Grande do Sul (RS). O RS abrange uma área total de 281.730,223 km² (IBGE Estados, 2013) e está compreendido entre as latitudes $33^{\circ} 43^{\prime} \mathrm{S}$ e $27^{\circ} 05^{\prime} \mathrm{S}$ e as longitudes $49^{\circ} 42^{\prime} \mathrm{W}$ e $57^{\circ} 40^{\prime} \mathrm{W}$.

\section{Metodologia}

Os dados utilizados nesse trabalho correspondem aos dados altimétricos derivados dos modelos digitais do terreno do SRTM e do ASTER GDEM e os programas utilizados para o cálculo da CT foram o TC2DFTPL e o TCFOUR.

O programa em FORTRAN77, chamado TC2DFTPL, desenvolvido por Li e Sideris (1994), calcula a correção do terreno via 2D-FFT. Três arquivos de saída são produzidos pelo programa: o primeiro é a correção do terreno a ser aplicada nos dados gravimétricos considerando o modelo topográfico como tendo a massa concentrada num prisma; o segundo é a correção do 
terreno a ser aplicada nos dados gravimétricos considerando o modelo topográfico de massa linear e o terceiro arquivo faz uma média entre os dois arquivos de saída anteriores. Nesse trabalho, considerou-se o modelo topográfico como tendo a massa concentrada num prisma.

O programa TCFOUR é um programa da IAG (International Geoid School) e faz parte do conjunto GRAVSOFT (Forsberg, 2009, apud Jamur e Freitas, 2012). Esse programa utiliza dois modelos digitais de elevação com resoluções diferentes para o cálculo da correção do terreno. O primeiro, de maior resolução, é responsável pela maior parte do efeito do terreno sobre a grandeza do campo da gravidade devido à proximidade do ponto de cálculo, sendo considerado até um raio $R_{0}$. O segundo, de menor resolução, é chamado de modelo de referência, que funciona como um filtro passa alta (Jamur e Freitas, 2012). O programa TCFOUR também calcula a correção do terreno via FFT e o resultado é mostrado na forma de um modelo digital. Nesse trabalho, foram utilizados modelos digitais de elevação com as seguintes resoluções: $10 \mathrm{~km}, 5 \mathrm{~km}, 2 \mathrm{~km}$ e $1 \mathrm{~km}$, extraídos dos MDTs do SRTM (com resolução espacial de $90 \mathrm{~m}$ ) e do ASTER (com resolução espacial de $30 \mathrm{~m}$ ), utilizando um $R_{0}=999 \mathrm{~km}$. A resolução dos modelos de referência utilizadas foi de $20 \mathrm{~km}$, também extraídas dos MDTs do SRTM e do ASTER.

Nos programas TC2DFTPL e TCFOUR foram calculadas as correções do terreno utilizando os MDTs do SRTM ( $1^{\underline{a}}$ etapa) e do ASTER ( $2^{\underline{a}}$ etapa) nas resoluções de $10 \mathrm{~km}, 5 \mathrm{~km}, 2 \mathrm{~km}$ e $1 \mathrm{~km}$. Na $1^{\text {a }}$ etapa, para o cálculo da CT no programa TC2DFTPL, foi necessário dividir a área do RS apenas para o modelo na resolução de $1 \mathrm{~km}$, assim a área do $\mathrm{RS}$ foi dividida em quatro partes. Já para o cálculo da CT no programa TCFOUR não foi necessário dividir a área do RS em nenhum dos modelos. Na $2^{\underline{a}}$ etapa, para o cálculo da CT no programa TC2DFTPL foi necessário dividir a área do RS para os modelos nas resoluções de $2 \mathrm{~km}$ e $1 \mathrm{~km}$, assim a área do RS foi dividida, respectivamente, em duas e nove partes. Já para o cálculo da CT no programa TCFOUR foi necessário dividir a área do RS apenas para o modelo de $1 \mathrm{~km}$, assim a área do RS foi dividida em quatro partes.

Pode-se notar que o programa TCFOUR apresentou efeito de borda para a área de estudo (Figura 6) e para as subdivisões necessárias da área de estudo, ao contrário do programa TC2DFTPL que não apresentou efeito de borda em nenhuma situação. Para efeito de comparação entre as escalas de variação da correção do terreno, calculadas pelos dois programas, o efeito de borda foi desconsiderado.

Após, comparou-se a CT calculada pelo programa TC2DFTPL utilizando os MDTs do SRTM nas resoluções de $5 \mathrm{~km}, 2 \mathrm{~km}$ e $1 \mathrm{~km}$ com a CT calculada por esse programa utilizando o MDT na resolução de $10 \mathrm{~km}$. Fezse o mesmo para as CTs calculadas pelo programa TCFOUR. Após, comparou-se a CT calculada por ambos os programas utilizando os MDTs do SRTM nas resoluções de $10 \mathrm{~km}, 5 \mathrm{~km}, 2 \mathrm{~km}$ e $1 \mathrm{~km}$. A comparação das CTs calculadas utilizando os MDTs gerados a partir dos dados ASTER foi feita da mesma forma. Assim, as comparações citadas anteriormente foram realizadas novamente, mas agora com os dados provenientes do ASTER.

\section{Resultados}

Através da análise da disposição espacial da correção do terreno, percebeu-se que as diferenças entre as CTs para o modelo de menor resolução $(10 \mathrm{~km})$ e os de maior resolução $(5 \mathrm{~km}, 2 \mathrm{~km}$ e $1 \mathrm{~km})$ concentram-se na região da Serra.

As correções do terreno calculadas pelos dois programas, utilizando os dados do SRTM e do ASTER, para o modelo de $10 \mathrm{~km}$ são iguais, pois não foi identificada nenhuma diferença maior do que 1 (um) mgal. Utilizando os dados do SRTM, notou-se que as CTs calculadas pelos dois programas para o modelo de 5 $\mathrm{km}$ são iguais, onde as diferenças concentram-se no limite nordeste do Estado (Figura 1). No entanto, as CTs calculadas por ambos os programas com os dados do ASTER para o modelo de $5 \mathrm{~km}$ apresentaram diferenças significativas entre elas, concentrando-se na região da Serra (Figura 2). Verificou-se também que as diferenças entre os modelos de $2 \mathrm{~km}$ e $1 \mathrm{~km}$, utilizando tanto os dados do SRTM quanto do ASTER, são significativas e concentram-se também na região da Serra (Figuras 3, 4, 5 e 6). Na Figura 6 pode-se perceber também o efeito de borda nas subdivisões feitas na área de estudo para o modelo de $1 \mathrm{~km}$.

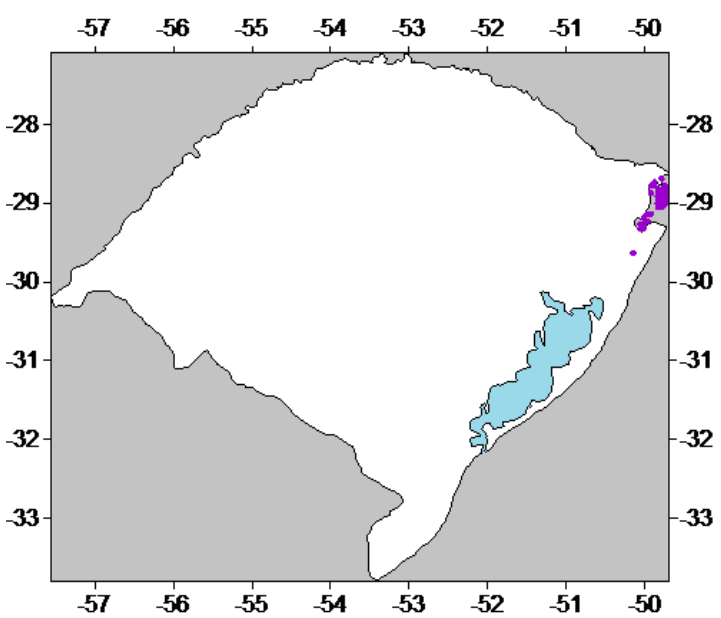

Figura 1 - TC2DFTPL $x$ TCFOUR_SRTM: $5 \mathrm{~km} \times 5 \mathrm{~km}$ 


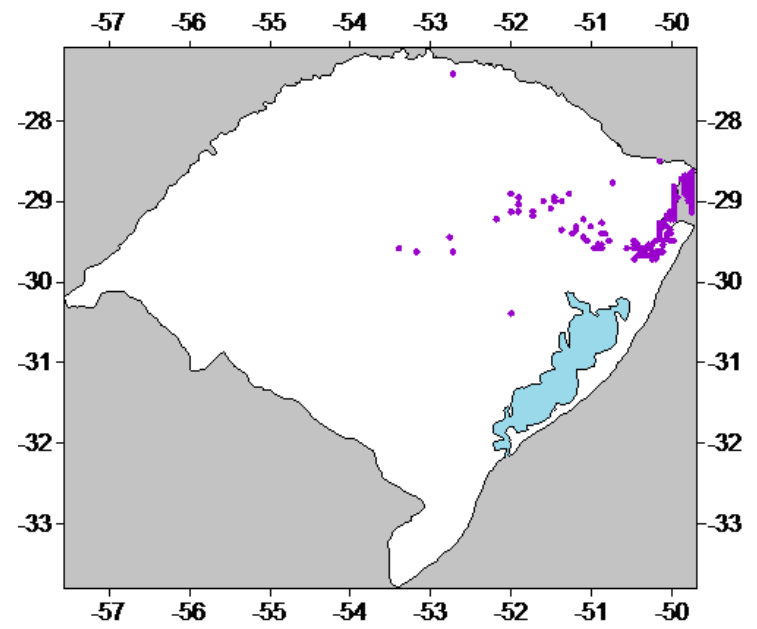

Figura 2-TC2DFTPL x TCFOUR_ASTER: $5 \mathrm{~km} \times 5 \mathrm{~km}$

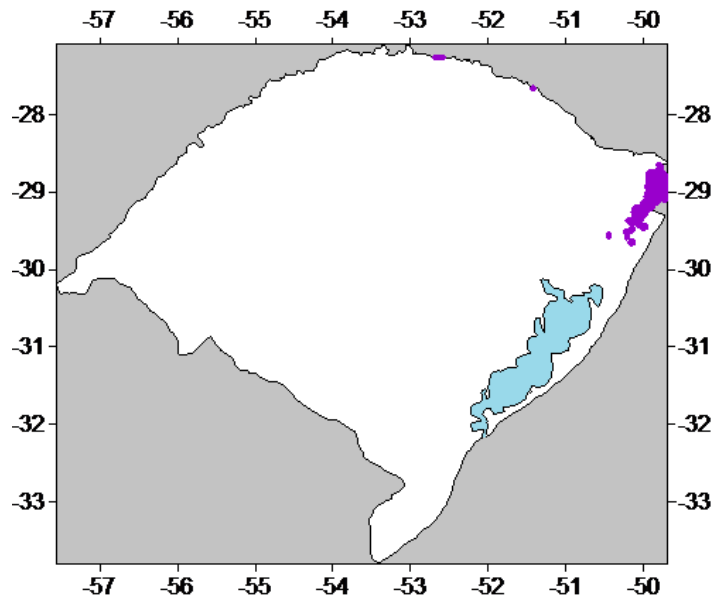

Figura 3-TC2DFTPL $x$ TCFOUR_SRTM: $2 \mathrm{~km} \times 2 \mathrm{~km}$

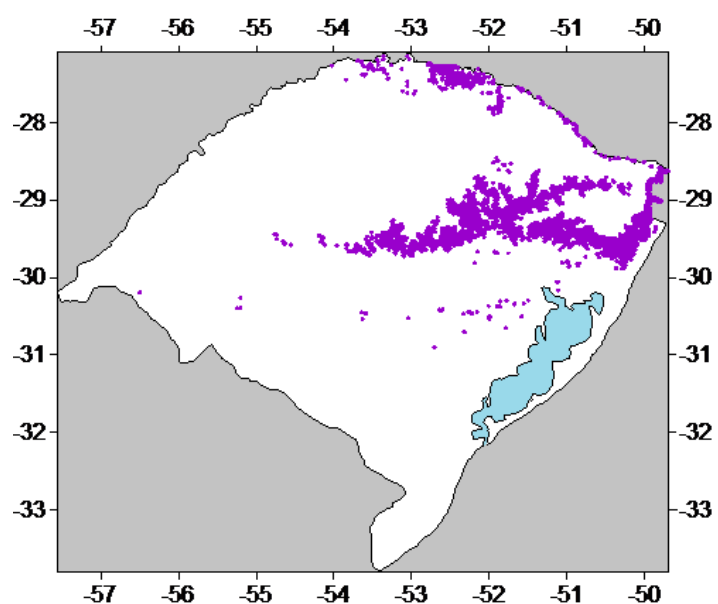

Figura 4 - TC2DFTPL $\times$ TCFOUR_SRTM: $1 \mathrm{~km} \times 1 \mathrm{~km}$

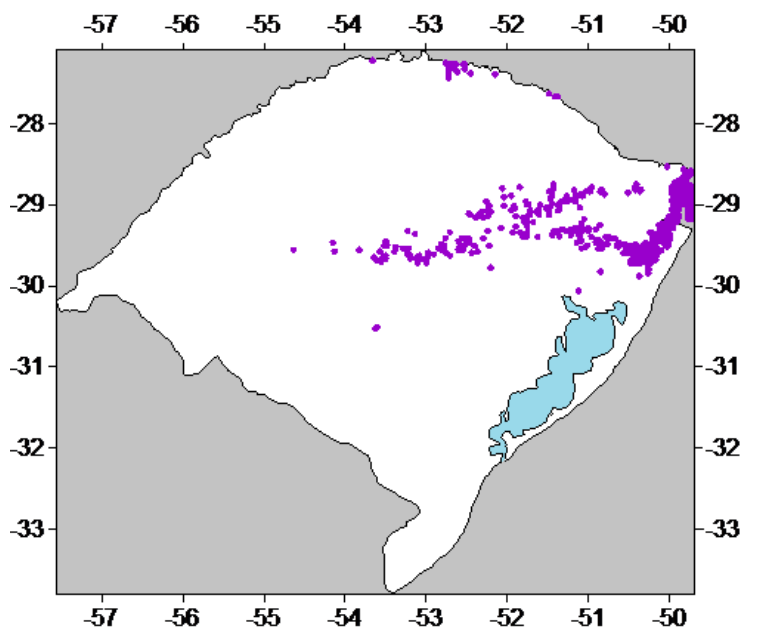

Figura 5-TC2DFTPL x TCFOUR_ASTER: $2 \mathrm{~km} \times 2 \mathrm{~km}$

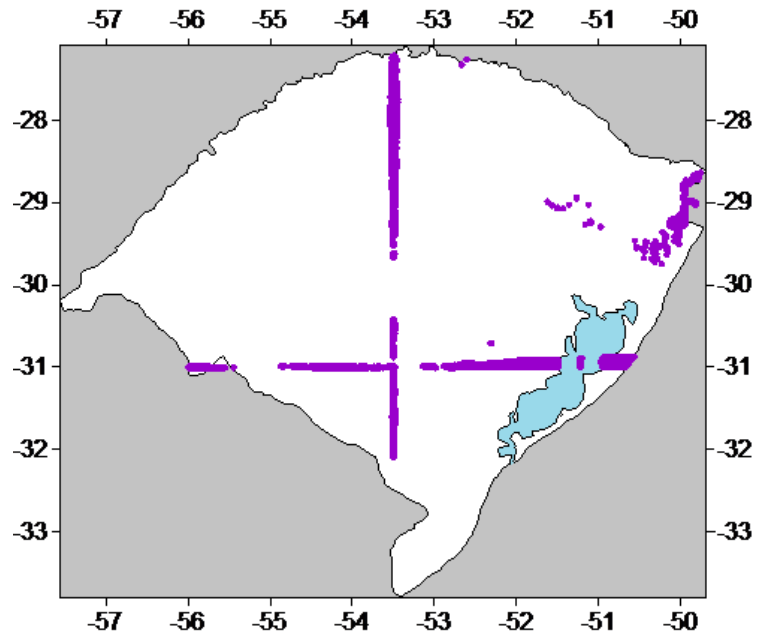

Figura 6-TC2DFTPL $x$ TCFOUR_ASTER: $1 \mathrm{~km} \times 1 \mathrm{~km}$

\section{Discussão e Conclusões}

As escalas de variação da correção do terreno calculadas pelos programas TC2DFTPL e TCFOUR, utilizando os dados do SRTM e do ASTER podem ser verificadas no Quadro 1.

Quadro 1 - Escalas de variação da Correção do Terreno (CT)

\begin{tabular}{|c|c|c|c|}
\hline \multirow{4}{*}{ Programa } & $\begin{array}{c}\text { Resolução } \\
\text { do MDT } \\
\text { (km) }\end{array}$ & \multicolumn{2}{|c|}{$\begin{array}{c}\text { Escala de variação da CT } \\
\text { (mgal) }\end{array}$} \\
\cline { 3 - 4 } & & $\begin{array}{c}\text { Dados } \\
\text { SRTM }\end{array}$ & $\begin{array}{c}\text { Dados } \\
\text { ASTER }\end{array}$ \\
\hline \multirow{4}{*}{ TC2DFTPL } & 10 & 0 a 7,0 & 0 a 6,4 \\
\cline { 2 - 4 } & 5 & 0 a 11,0 & 0 a 10,5 \\
\cline { 2 - 4 } & 2 & 0 a 20,0 & 0 a 13,4 \\
\hline \multirow{3}{*}{ TCFOUR } & 1 & 0 a 14,8 & 0 a 13,7 \\
\cline { 2 - 4 } & 10 & 0 a 3,2 & 0 a 3,5 \\
\cline { 2 - 4 } & 5 & 0 a 6,5 & 0 a 6,0 \\
\cline { 2 - 4 } & 2 & 0 a 12,4 & 0 a 13,8 \\
\hline
\end{tabular}


Analisando o Quadro 1 nota-se que existe uma relação diretamente proporcional entre a resolução do modelo digital de terreno utilizado no cálculo da correção do terreno e o valor da correção a ser aplicada nos dados gravimétricos: à medida que a resolução do MDT utilizado aumenta, a CT a ser aplicada nos dados gravimétricos também aumenta. No entanto, isso não ocorre quando se faz necessário dividir a área de estudo (ver Quadro 2) para que se torne possível o cálculo da correção do terreno pelos programas. Assim, concluiu-se que, nos casos em que seja necessário subdividir a área de estudo, o valor da correção do terreno calculada sofrerá suavizações (principalmente as correções calculadas pelo programa TC2DFTPL, como pode ser verificado através do Quadro 1). A escala de variação da CT para o MDT com resolução de $1 \mathrm{~km}$ do ASTER, calculado pelo programa TCFOUR, apresenta seus valores comprometidos devido à presença do efeito de borda nas subdivisões da área.

No Quadro 2 é possível verificar para quais resoluções de MDTs foi necessário compartimentar a área de estudo.

Quadro 2 - Necessidade de compartimento da área de estudo para o cálculo da CT pelos programas.

\begin{tabular}{|c|c|c|c|}
\hline \multirow{4}{*}{ Programa } & $\begin{array}{c}\text { Resolução } \\
\text { do MDT } \\
\text { (km) }\end{array}$ & \multicolumn{2}{|c|}{$\begin{array}{c}\text { Divisão da área de } \\
\text { estudo }\end{array}$} \\
\cline { 2 - 4 } & SRTM & ASTER \\
\hline \multirow{4}{*}{ TC2DFTPL } & 10 & Não & Não \\
\cline { 2 - 4 } & 5 & Não & Não \\
\cline { 2 - 4 } & 2 & Não & Sim \\
\cline { 2 - 4 } & 1 & Sim & Sim \\
\hline \multirow{4}{*}{ TCFOUR } & 10 & Não & Não \\
\cline { 2 - 4 } & 5 & Não & Não \\
\cline { 2 - 4 } & 2 & Não & Não \\
\cline { 2 - 4 } & 1 & Não & Sim \\
\hline
\end{tabular}

Ao final de todas as avaliações pode-se concluir que os programas TC2DFTPL e TCFOUR são praticamente idênticos e que ocorrem suavizações nos valores da CT calculadas a partir do uso de MDTs com resolução de $5 \mathrm{~km}$, pois para resoluções maiores $(2 \mathrm{~km}$ e $1 \mathrm{~km}$ ) ambos os programas não detectam a melhora do MDT. Isso pode ser considerado um problema quando o objetivo é determinar geoides locais.

No entanto, pode-se aconselhar a utilização do programa TC2DFTPL para o cálculo da correção do terreno, ao invés do programa TCFOUR (apesar deste apresentar uma capacidade maior de processamento de dados), pois o programa TC2DFTPL não apresenta efeito de borda. Porém, a sua utilização não é indicada quando a área que está sendo estudada é muito extensa e for necessário compartimentar a área, pois, como pode ser verificado nos Quadros 1 e 2, as subdivisões da área de estudo ocasionam suavizações nos valores da CT calculadas pelo programa.

Concluiu-se também que a incompatibilidade das escalas de variação da CT dos modelos derivados do SRTM e do ASTER, com resoluções de $2 \mathrm{~km}$ e $1 \mathrm{~km}$, se deva à necessidade de subdividir a área de estudo para essas resoluções e também às diferenças altimétricas entre os dois MDTs, diferenças essas que são mais evidentes nas regiões de maiores altitudes, como é o caso da região da Serra do RS.

\section{Agradecimentos}

Este trabalho faz parte de um projeto mais amplo sobre a determinação e avaliação do geóide no Estado do Rio Grande do Sul. Esse projeto conta com o apoio do CNPQ na forma de fomento a projeto de pesquisa, através do apoio financeiro recebido em forma de Bolsa de Iniciação Científica PIBIC/CNPq-UFRGS.

\section{Referências}

IBGE Estados. 2013. Rio Grande do Sul. Disponível em: <http://www.ibge.gov.br/estadosat/perfil.php?sigla=rs>. Acesso em 08/11/2013.

Jamur, K. P.; Pereira, R. A. D.; de Freitas, S. R. C.; Miranda, F. D. A.; Ferreira, V. G. 2010. Avaliação da correção gravimétrica do terreno obtida de modelos digitais de elevação associados ao SGB e ao EGM2008. III Simpósio Brasileiro de Ciências Geodésicas e Tecnologias da Geoinformação. p. 001-005.

Jamur, K. P.; de Freitas, S. R. C. 2012. Estudo de alternativas para combinar altimetria e gravimetria por satélites para uso em regiões de baixa cobertura gravimétrica convencional. III Simpósio Brasileiro de Geomática. p. 280-295.

Li, Y. C.; Sideris, M. G.1994. Improved gravimetric terrain corrections. Geophysical Journal International, v. 119, p. 740-752.

Matos, A. C. O. C. 2005. Implementação de modelos digitais de terreno para aplicações na área de geodésia e geofísica na América do Sul. São Paulo. 355p. Tese de Doutorado em Engenharia, Escola Politécnica da Universidade de São Paulo.

Sideris, M. G. 1985. A fast fourier transform method of computing terrain corrections. Manuscripta Geodaetica, v. 10, p. 66-73. 